Part of Journal of Research of the National Bureau of Standards, Volume 21, December 1938

\title{
ON THE ACCURACY OF RADIO FIELD-INTENSITY MEASUREMENT AT BROADCAST FREQUENCIES
}

\author{
By Harry Diamond, Kenneth A. Norton,* and Evan G. Lapham
}

\begin{abstract}
This paper presents a discussion of errors commonly encountered in radio fieldintensity measuring sets operating at broadcast frequencies. Data are given to indicate the magnitudes of the different errors existing in typical commercial measuring sets, and an estimate is presented of the probable accuracy of measurement attained. Of the several errors, the one resulting from the simplifying assumption that a loop antenna has the same voltage step-up for a distributed induced voltage as for a lumped voltage introduced at its center is often the most serious. An analysis is given to show how this error arises and an expression is derived for taking it into account. A method for eliminating this error, suggested by Baker and Huxley, is discussed, and data are given illustrating the efficacy of this method. Other means for limiting this error are also discussed.
\end{abstract}

\section{CONTENTS}

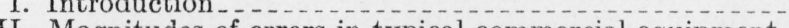

II. Magnitudes of errors in typical commercial equipment

III. Analysis of effect of distributed capacitance of loop antenna _._. 799

IV. Analysis of experimental data on distributed-capacitance effect _. _ 806

V. $\Delta C$ method for eliminating the distributed-capacitance effect _.... 807

VI. Untuned loop antenna_._.

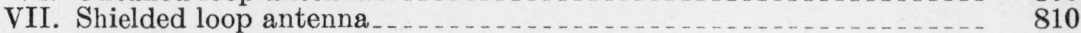

VIII. Precautions for attaining maximum accuracy _... .

IX. Conclusions . ................. 817

\section{INTRODUCTION}

The increasing interest in the quantitative determination of the service area of broadcast stations, coupled with a more rigorous approach to the derivation of radio-wave attenuation formulas, has resulted in a twofold interest in the accuracy of radio field-intensity measurements at broadcast frequencies. This interest has been reflected in the increased number of measuring sets calibrated at the National Bureau of Standards during the past few years. In general, the sets tested employed one of three methods of measurement and were found to be subject to typical errors, characteristic of these methods. This paper presents the results of a critical study of the magnitude of the errors encountered with the three different methods; consideration of these errors is restricted to operating frequencies below $1,600 \mathrm{kc} / \mathrm{s}$, and to field intensities ranging from $100 \mu \mathrm{v} / \mathrm{m}$ to 0.1 $\mathrm{v} / \mathrm{m}$.

\footnotetext{
* Associate Engineer, Federal Communications Commission.
} 
The nature of the errors likely to be involved may best be understood from consideration of the manner in which the measurement of the field voltage induced in the loop antenna is made in the three methods. In one method, the first detector of a superheterodyne receiver, used in conjunction with a voltage-attenuator connected between the detector and the intermediate amplifier, constitutes the voltage determining means. The detector is first calibrated at highvoltage level in a low-frequency calibrating circuit. A comparison is next made between the voltages developed across one-half of the loop antenna by the field being measured and by a local voltage of identical frequency inserted at the center of the loop antenna and adjusted to give the same current in the plate circuit of the detector as that given by the low-frequency calibrating voltage. The voltage step-up, or $Q$-factor, of the loop antenna is then measured by a similar comparison between the local voltage applied directly to the detector input and the voltage it develops across one-half the loop antenna by virtue of resonance. From these two measurements, the field voltage induced in the antenna may be computed.

In the second method of measurement, the voltage-determining means consists of a voltage generator and attenuator for introducing at the center of the loop antenna a known voltage of the same radio frequency as the field voltage. Equality of the two voltages is determined by using the receiving set as an uncalibrated vacuum-tube voltmeter, usually connected across one-half of the loop antenna. The third method of measurement differs from the second in that the calibrating voltage is of fixed value (high level) and is compared with the induced field voltage by means of the first detector of a superheterodyne receiver used in conjunction with a voltage attenuator connected between the first detector and the intermediate amplifier. In a commercial equipment employing this method the gap between successive steps of the voltage attenuator is spanned by a linear outputindicating system comprising a half-wave rectifier and indicating: meter.

It will be evident that the following factors may influence the correctness of the measured field voltage in one or all of the three methods considered: (a) incorrect calibrating voltage; (b) nonlinearity of the detector and of the output indicating system; (c) incorrect voltage-attenuator design; (d) stray voltages induced in the loop antenna by the calibrating oscillator or from other portions of the set; (e) regeneration in various portions of the set; and (f) inaccurate introduction of the calibrating voltage because of unbalance of the loop antenna. The accuracy of field-intensity measurements will be affected by the cumulative error in the voltage determination and, in addition, by two other possible errors which we shall term: $(\mathrm{g})$ the proximity effect, and (h) the distributed-capacitance effect. The proximity effect consists in the distortion of the measured field caused by the set container or by other objects near the loop antenna; for example, a portion of the automobile in which the set may be installed. The distributed-capacitance effect consists in the difference produced by the distributed capacitance of the loop antenna in the effective voltage step-up of the tuned loop-antenna circuit for the distributed field voltage and for the lumped calibrating voltage.

The distributed-capacitance effect appears to have been given scant consideration in the design of commercial apparatus. The error 
in the measured value of the $Q$-factor, caused by the distributed capacitance, is a function of the ratio of the operating frequency to the natural frequency of the antenna. In some commercial sets, in which the loop antennas operate near their natural frequencies when in the upper portions of their frequency ranges, the distributed-capacitance error is of the order of 15 percent. An analysis of this error is given in the paper together with a derived expression for the correction factor to be applied in order to compensate for it. The computed factor agrees well with experimental results.

\section{MAGNITUDES OF ERRORS IN TYPICAL COMMERCIAL EQUIPMENT}

In this section are presented data on the magnitudes of the several errors as measured in commercial equipment typical of the three methods of field-intensity measurement described. Based on these data, an estimate is given of the absolute accuracy of apparatus representative of each of the three methods.

In the measurement of several sets of a commercial type employing the first method, the following errors were detected. An error of up to 6 percent was caused by harmonics in the 1,000-cycle calibrating voltage and by a change in the detector grid bias (from its operating value), when the calibrating circuit was turned "on." An error of up to 16 percent was caused by nonlinearity of the detector; the departure from linearity was found to vary with operating frequency in several sets because of the variable voltage introduced in the detector platecircuit by the heterodyne oscillator. In one set, faulty neutralization of the detector (produced by an unbalance in the modulating circuit due to a poor electron tube) caused an error of up to 5 percent. Leakage from the local oscillator was of negligible order unless an attempt was made to use low-level calibration of the detector; in the latter case an error of up to 5 percent was produced in the measurement of the $Q$-factor, depending upon the orientation of the loop antenna. An error of up to 8 percent was introduced by the effect of the distributed capacitance of the antenna upon the measurement of its $Q$-factor; in this particular design the limited operating frequency range of each loop antenna (2.2 to 1) resulted in limiting the maximum value of this error to 8 percent. The distortion of the field by the set container was found to be responsible for an error ranging from 0 to 10 percent as the orientation of the loop antenna was varied from a plane perpendicular to the longitudinal axis of the container to a plane containing this axis. Errors of types (c) and (f) were found to be negligible.

In estimating the absolute accuracy of measurement for this method, consideration must be given to the difference in sign of several of the errors and the variation of several with the operating frequency. The absolute accuracy appears to be not greater than 20 percent for stock equipment. However, an accuracy of 5 percent may be attained if the following precautions are taken: correction of possible maladjustments of set; positioning of the set to provide for optimum orientation of the loop antenna or use of an extension fitting to raise the loop antenna appreciably above the set: measurement and application of a correction factor for the nonlinearity of the first detector; and application of a correction factor for the distributedcapacitance effect. 
The accuracy of the second method of field-intensity measurement discussed, depends largely upon the accuracy of the calibrated voltage generator. Since commercial equipment of this type is available with an accuracy of \pm 3 percent up to $3,000 \mathrm{kc} / \mathrm{s}$, this method combines simplicity of manipulation with accuracy of the voltage-determining means. Other possible sources of error with this method are (d), (e), (f), (g), and (h) of the enumeration in section I. The error of type (h) is present in this method, even though a $Q$-factor measurement is not explicitly made, because the distributed field voltage and the lumped calibrating voltages are stepped-up differently by resonating the loop antenna prior to comparison.

In a number of sets employing this method which were tested, errors of types (d), (e), and (f) were generally found of negligible order. In several sets, mounted on automobiles with the loop antennas extending above the top, errors of type $(\mathrm{g})$ were up to 20 percent in magnitude and varied with the orientation of the loop antenna with respect to the automobile. One set which employed a single loop antenna to cover a frequency range of from 550 to $1,550 \mathrm{kc} / \mathrm{s}$ had an error of type (h) equal to 12 percent at the upper frequency. In general, sets of this type have a probable over-all absolute accuracy of not better than 15 percent. (This neglects the proximity error caused by installation in an automobile.) When a correction factor is applied for the effect of distributed capacitance of the loop antenna, a probable absolute accuracy of about 5 percent appears reasonable.

The sets tested, representative of the third method of field-intensity measurement, were of identical manufacture. The following errors were detected. Errors in the calibrating voltage ranged up to 5 percent. One set showed an error caused by nonlinearity of the first detector up to 5 percent; the other sets were free from this error. The voltage attenuator was in general accurate to within the limits of measurement, that is, 2 percent. However, individual steps of the attenuator were found to be in error up to 10 percent in two of the sets. The output indicating system was in general linear within 2 percent, provided that the lower sixth of the indicator scale was not employed. However, in two sets, aging of the output meter produced errors up to 8 percent. Errors of the types (d) and (e) were found to be of negligible order. Incorrect setting of the antenna balancing condenser in one set produced an error of type (f) ranging up to 6 percent at $1,500 \mathrm{kc} / \mathrm{s}$. This type of equipment employs a single loop antenna to cover the frequency range of from 550 to $1,550 \mathrm{kc} / \mathrm{s}$ and hence is subject to a material distributed-capacitance error at the upper frequencies; the magnitude of this error was found to be 15 percent at $1,500 \mathrm{kc} / \mathrm{s}$ in all of the sets tested.

The over-all absolute accuracy of this equipment may be estimated at not better than 20 percent. After application of a correction factor for the distributed-capacitance effect, the probable accuracy becomes about 10 percent. An absolute accuracy of 5 percent may be attained by correcting for such errors as may be present in the calibrating voltage, in the introduction of the calibrating voltage, in possible nonlinearity of the detector, in individual steps of the voltage attenuator, and in the output indicating system. 


\section{ANALYSIS OF EFFECT OF DISTRIBUTED CAPACITANCE OF LOOP ANTENNA}

The importance of the difference in the $Q$-factor of the loop antenna for a distributed induced voltage and for a lumped voltage at its center has been indicated in the previous section. The availability of an exact correction factor which could be employed to take this effect into account would increase the absolute accuracy of commercial radio field-intensity measuring sets to an appreciable degree. An analysis of the underlying phenomenon leading to the derivation of the correction factor follows.

As already indicated, the difference in the $Q$-factor arises from the distributed capacitance of the loop antenna. Because of the distributed capacitance, the current is different in different portions of the loop antenna. The nature of the effect of the distributed capacitance upon the current distribution in a tuned loop antenna (and upon the distribution of the equivalent effective resistance) was given in several papers by Breit ${ }^{1}$ in 1921 and 1922 .

Breit showed that the current distribution at resonance is independent of the distribution of voltage and is the same as if the system were nondissipative. The latter point leads to the conclusion that the current distribution is also independent of resistances introduced at various points along the loop antenna and enabled Breit to measure the current distribution in several loop antennas by the resistancevariation method. In an unpublished thesis submitted to the University of Minnesota in 1928, Bailey ${ }^{2}$ proved this conclusion experimentally.

Proceeding with Breit's theory, the currents at any two points, 1 and 2 , along a loop antenna are related by eq 1 below, which is based on the equality of power at the two points. (The resistances $R_{1}$ and $R_{2}$ may be defined as the equivalent resistances at points 1 and 2 ).

$$
\frac{i_{1}}{i_{2}}=\frac{\sqrt{R_{2}}}{\sqrt{R_{1}}}
$$

Placing $e_{2}=R_{2} i_{2}$, and substituting for $i_{2}$ in eq 1 , we have

$$
i_{1}=\frac{e_{2}}{R_{2}} \cdot \sqrt{\frac{R_{2}}{R_{1}}}=\frac{e_{2}}{\sqrt{R_{1} R_{2}}} .
$$

Hence, the relation between the current $\left(i_{0}\right)$ which enters the effective tuning condenser as a result of a lumped voltage $\left(e_{m}\right)$ inserted at the center of the loop antenna is given by eq 3 ,

$$
i_{0}=\frac{e_{m}}{\sqrt{R_{0} R_{m}}} .
$$

In this equation, $R_{0}$ is the equivalent resistance of the loop antenna at its grid end and $R_{m}$ is the equivalent resistance at its center. (The significance of the terms $i_{0}$ and $e_{m}$ will appear from figure 1(a), which corresponds substantially to the circuit arrangement employed in each

$1 \mathrm{G}$. Breit, The distributed capacitance of inductance coils, Phy. Rev. 17, 649 (1921).

G. Breit, High-frequency resistance of inductance coils. BS Sci. Pap. 17, 569 (1922) S430.

2 Stuart L. Bailey, The characteristics of the coil aerial as used with radio signal intensity measuring apparatus. Thesis for degree of Master of Science, submitted to University of Minnesota (June 1928). 
of the three methods of field-intensity measurement discussed.) For a uniformly distributed voltage $\left(e_{x} \cdot d x\right)$ induced per elementary length $(d x)$ of wire

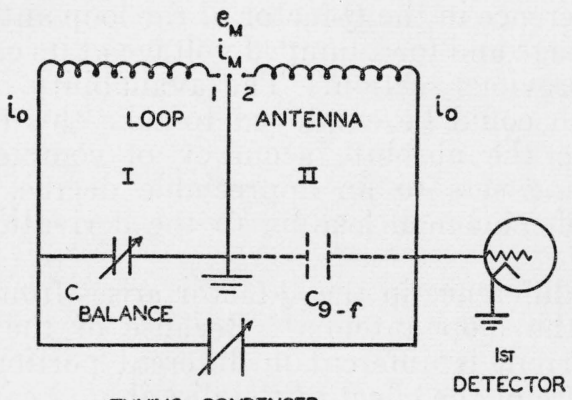

TUNNG CONDENSER

(a)

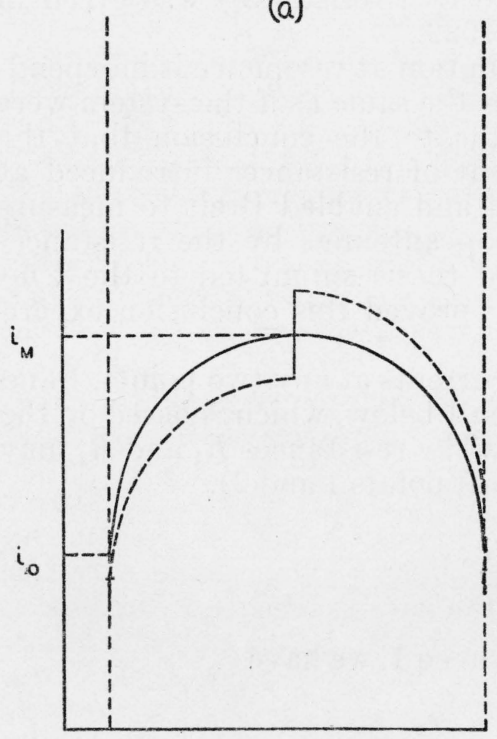

TURNS ALONG LOOP ANTENNA

(b)

FigURE 1.--Arrangement of balanced loop antenna in the methods of field-intensity measurement considered, and current distributions for two conditions of antenna balance.

$$
i_{0}=\frac{1}{\sqrt{R_{0}}} \int \frac{e_{x} \cdot d x}{\sqrt{R_{x}}}
$$

where $R_{x}$ is the equivalent resistance at the point $x$ along the wire.

Now, in each of the methods of measuring field intensity discussed in the foregoing section, $i_{0}$ corresponding to the lumped and distributed voltages is adjusted to equality and the assumption is made that for this condition, writing $h_{e}$ for the effective height of the loop antenna and $\epsilon$ for the field intensity

$$
e_{m}=\left[\int e_{x} \cdot d x=h_{e} \epsilon\right]
$$

instead of the actual relationship from eq 3 and 4, namely,

$$
e_{m}=\int e_{x} \sqrt{\frac{R_{m}}{R x}} \cdot d x
$$

Therefore, a certain factor must be applied to the value of $e_{m}$ in order to determine the true value of the induced voltage. This correction factor, derived from the approximate expression (eq 5a) and the correct expression (eq 5 b), is defined by $F$ in the following equation:

$$
\frac{1}{F}=\frac{\int e_{x} \cdot \sqrt{\frac{R_{m}}{R_{x}}} \cdot d x}{\int e_{x} \cdot d x},
$$

which may be written by using eq 1 :

$$
\frac{1}{F}=\frac{\int e_{x} \cdot \frac{i_{x}}{i_{m}} \cdot d x}{\int e_{x} \cdot d x}
$$


Now, if the induced voltage per elementary length is assumed independent of $x$ (that is, is uniformly distributed), we have

$$
\frac{1}{F}=\frac{e_{x} \cdot \frac{1}{i_{m}} \int i_{x} \cdot d x}{e_{x} \int d x}=\frac{i_{a v e}}{i_{m}}
$$

and

$$
F=\frac{i_{m}}{i_{a v e}}
$$

The correction factor is seen to be intimately tied up with the current distribution along the loop antenna. The factor $F$ differs from unity to the extent that the distributed capacitance of the loop antenna causes the current at resonance to vary throughout the antenna.

So far in our analysis, no consideration has been given to the actual current distribution along the loop antenna. To evaluate the correction factor an exact knowledge of the current distribution is required. Referring to figure 1(b), the solid curve shows qualitatively the current distribution when the loop antenna is exactly balanced. The symmetry of the distribution curve is lost if any unbalance of the loop antenna occurs. Thus, the dotted curve corresponds to the current distribution when the balancing condenser is reduced below its value for exact balance. It should be emphasized that the distribution curves shown apply independently of the voltage distribution; the latter may be uniformly distributed or introduced as a lumped voltage at points 1 or 2 , figure 1 (a). In the following derivations, exact symmetry of the current-distribution curve is assumed and, hence, exact antenna balance. The effect of antenna unbalance will be treated later in this paper.

A summation relationship equivalent to eq 6 was derived by Bailey (see footnote 2), in an extension of Breit's work carried on to derive an expression for the correction factor with which we are dealing. Bailey chose to work with resistance distribution because of the possibility of measuring the effective resistance directly. He found that the resistance distribution could be represented by an inverse parabolic curve and derived the following expression

$$
R_{x}=\frac{R_{m}}{1-\left(1-\frac{R_{m}}{R_{0}}\right) x^{2}},
$$

where $x=2 n / N, N$ being the total number of turns in the loop antenna and $n$ indicating the number of turns from the center. By measuring the terminal resistances of a loop antenna at several frequencies and averaging $\sqrt{\left(R_{m} / R_{x}\right)}$ along only the vertical portions of the loop antenna to take account of the fact that the field voltage is not actually uniformly distributed along the entire loop antenna, Bailey obtained several values for the correction factor. Actually, the error introduced by assuming the voltage uniformly distributed is small for even a twoturn loop antenna of any shape and becomes negligible as the turns are increased to about six. Since Bailey used a six-turn loop antenna, his 
factor may be calculated by using eq 6 and 10, with a negligible error, giving

$$
\frac{1}{F}=\frac{1}{2}\left\{\sqrt{\frac{R_{m}}{R_{0}}}+\frac{\sin ^{-1} \sqrt{1-\frac{R_{m}}{R_{0}}}}{\sqrt{1-\frac{R_{m}}{R_{0}}}}\right\}
$$

However, the utility of eq 11 is limited in the practical case, since it is not usually convenient to measure either $R_{0}$ or $R_{m}$ in commercial field-intensity measuring sets. A second method of attack is made possible by considering the following relation derived by Breit

$$
\frac{i_{0}}{i_{a v e}}=1-\left(\frac{f}{f_{0}}\right)^{2}
$$

where $\left(f / f_{0}\right)$ is the ratio of the operating frequency to the natural frequency of the loop antenna. Assuming a current distribution to correspond with the inverse parabolic resistance distribution found by Bailey, it is possible to determine from eq 1,10 , and 12 the relationship between $i_{x}$ and $i_{m}$ as a function of the argument $\left(f \mid f_{0}\right)$ and thus from eq 8 to derive a general expression for $F$ in terms of $\left(f / f_{0}\right)$. Thus, we have the factor expressed in terms of an argument which may be readily measured. The relationship for the current distribution and the corresponding expression for $F$ are given, respectively, in the first line of the second and third columns of table 1.

Working independently, Baker and Huxley ${ }^{3}$ derived an expression for $F$ as a function of the ratio of the distributed capacitance to the tuning capacitance of the loop antenna on the basis that the loop antenna may be represented as a uniform transmission line. Expressions for the current distribution corresponding to this assumption and for the factor $F$ in terms of the argument $\left(f / f_{0}\right)$ are given, respectively, in the second line, second and third columns, of table 1.

Still another possible workable assumption is to proceed from eq 8 and 12, assuming a parabolic current distribution. Breit stated in his papers that this distribution corresponded approximately to his measured values. The derived expressions for current distribution and the factor $F$, corresponding to this assumption, are given, respectively, in the third line, second and third columns, of table 1.

However, none of the three relations for the correction factor was found to agree with many experimental determinations made in the process of calibrating field-intensity measuring sets at the National Bureau of Standards. An orderly examination of the data showed that the correction factor should be of the order of one-half that given by the transmission-line theory, and materially lower than given by the other two theories, particularly for the larger values of $\left(f / f_{0}\right)$. Accordingly, a further examination of the underlying theory was made. Studying Breit's analysis, it became apparent that his mathematics pointed to an elliptical current distribution even though he had suggested from his measured values that the distribution was approximately parabolic. An expression for $F$ derived on the basis of elliptical current distribution, was found to fit the data rather nicely. The

${ }^{3}$ W. G. Baker and L. G. H. Huxley, Correction to Field Strength Measurements with Loop Antennae. Radio Research Board of Australia Bulletin 47, Report 1, Melbourne (1931). 
expressions for the current distribution and for the correction factor, corresponding to this assumption of an elliptical current distribution, are given, respectively, in the fourth line, second and third columns, of table 1 .

It should be noted in connection with table 1, that the correction factors tabulated are based on an assumption of uniformly distributed field voltage along the wire of the loop antenna and hence are not exact. However, as has already been noted, the departure from exactness is quite small and decreases with an increasing number of turns in the loop antenna. In the case of the elliptical current distribution, a two-turn square-loop antenna mounted on a side would have as its exact correction factor the value

$$
F=\frac{1+\left(\frac{4}{\pi}-1\right) \alpha}{1+0.030 \alpha},
$$

while for a two-turn square-loop antenna mounted on a corner, the exact factor would be

$$
F=\frac{1+\left(\frac{4}{\pi}-1\right) \alpha}{1+0.020 \alpha}
$$

TABLE 1.-Expressions for current distribution and correction factor corresponding to the four assumptions considered

\begin{tabular}{|c|c|c|}
\hline Basic assumption & $\begin{array}{l}\text { Expression for current distribution } \\
\qquad\left(i_{x} / i_{m}\right)\end{array}$ & $\begin{array}{l}\text { Expression for correction } \\
\text { factor, } F\end{array}$ \\
\hline $\begin{array}{l}\text { Inverse parabolic distribu- } \\
\text { tion of resistance }\end{array}$ & $\sqrt{1-x^{2} \sin ^{2} \theta}$, where $\frac{\theta}{\sin \theta \cos \theta}=\frac{1+\alpha}{1-\alpha}$ & $\frac{1-\alpha}{\cos \theta}$, where $\frac{\theta}{\sin \theta \cos \theta}=\frac{1+\alpha}{1-\alpha}$ \\
\hline $\begin{array}{l}\text { Uniform transmission-line } \\
\text { theory }\end{array}$ & $\begin{array}{c}\cos \phi \cos \{(1-x) \phi\}+\sin \phi \sin \{(1-x) \phi\} \\
\text { where } \frac{\phi}{\tan \phi}=1-\alpha\end{array}$ & $\frac{\phi}{\sin \phi}$, where $\frac{\phi}{\tan \phi}=1-\alpha$ \\
\hline $\begin{array}{l}\text { Parabolic current distribu- } \\
\text { tion. }\end{array}$ & $1-\frac{3 \alpha}{2+\alpha} \cdot x^{2}$ & $1+\frac{1}{2} \cdot \alpha$ \\
\hline $\begin{array}{l}\text { Elliptical current distribu- } \\
\text { tion. }\end{array}$ & $\frac{1+\frac{4}{\pi} \cdot \frac{\alpha}{1-\alpha} \cdot \sqrt{1-x^{2}}}{1+\frac{4}{\pi} \cdot \frac{\alpha}{1-\alpha}}$ & $1+\left\{\frac{4}{\pi}-1\right\} \cdot \alpha$ \\
\hline
\end{tabular}

$\left[\alpha=\left(f / f_{0}\right)^{2}\right.$ and $\left.x=2 n / N\right]$

The factor rapidly converges to that given in the table, as the number of turns increases. Thus, for a four-turn square-loop antenna mounted on a side, the exact factor is

$$
F=\frac{1+\left(\frac{4}{\pi}-1\right)}{1+0.011 \alpha}^{\alpha}
$$

In figure 2 are presented plots of the factor $F$ as a function of $\left(f / f_{0}\right)$, corresponding to the four basic assumptions outlined. The four points of figure 2 designated by cross symbols correspond to 
data derived empirically by Bailey on the basis of resistance-distribution measurements. These should check exactly the graph corresponding to inverse parabolic resistance distribution; the slight

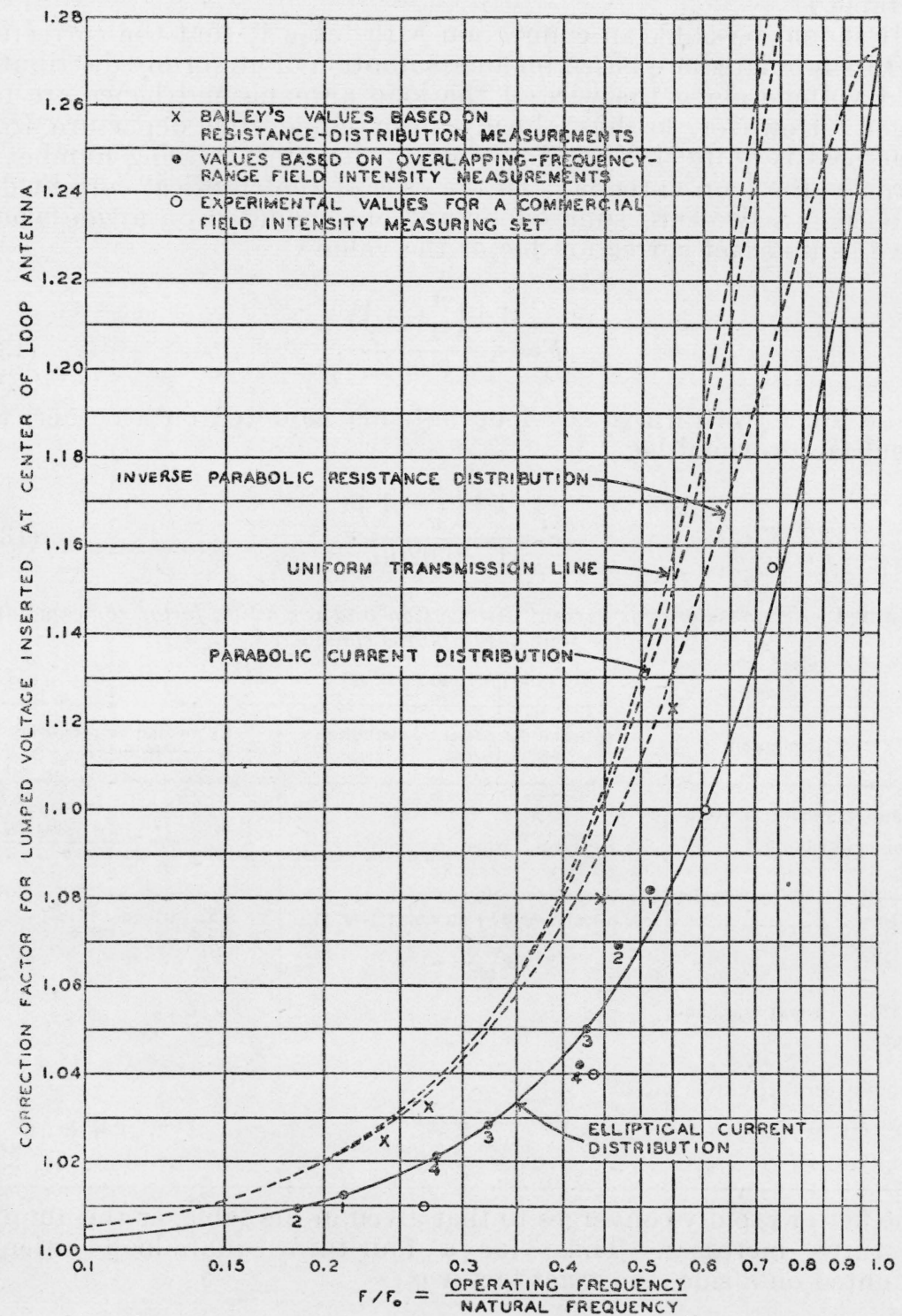

Figure 2.-Graphs showing the derived correction factors for the distributed-capacitance effect corresponding to four assumed distributions of current along the turns of the loop antenna.

The graph corresponding to elliptical current distribution agrees well with the experimental data.

departure is probably due to experimental errors in measurement. It will be noted from figure 2 that the assumption of elliptical current distribution yields values of $F$ which are materially lower than ob- 
tained from the other three theories. An analysis of experimental results to show that this is probably the correct factor will be given in the following section.

Before proceeding to the experimental results, it is of interest to consider the order of agreement of the several assumed current distributions with available published values. In figure 3 are plotted the four current distributions considered; the ordinate scale corresponds to the ratio of the current on specific turns of a loop antenna to the current at the center, while the abscissa scale corresponds to the turns number. The plot is carried out for a 14-turn loop antenna, since Breit published measured current values for such an antenna. Breit's values are shown by the open-circle symbols in figure 3 . It will be seen that the curve of elliptical current distribution conforms

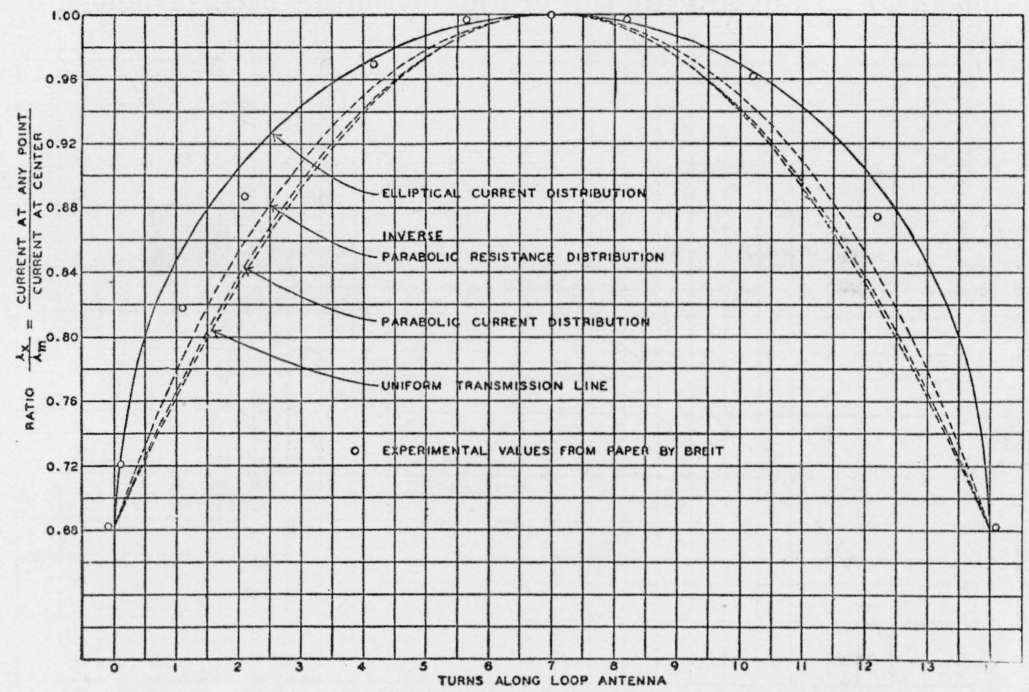

FIGURE 3.-Graphs showing the current distribution corresponding to the four assumptions treated in the theoretical analysis.

The open-circle symbols denote experimental values from Breit's papers.

most closely to the measured distribution. Further evidence for the probable correctness of the assumed elliptical current distribution is seen from figure 4 . In this figure are plotted graphs corresponding to three of the assumed distributions, showing the variation of the ratio of the current at the center of the loop antenna to the current at its ends as a function of the operating frequency. (Because of the close equivalence of the current distributions for the uniform transmission line theory and the inverse parabolic resistance theory, the graph for the former is not included in fig. 4.) The points having open-circle symbols denote the values of the ratio taken from Breit's published data, while the points having cross symbols are derived values based on Bailey's resistance measurements. It will be observed that the case for elliptical current distribution again agrees most closely with the experimental data. 


\section{ANALYSIS OF EXPERIMENTAL DATA ON DISTRIBUTED-CAPACITANCE EFFECT}

The ratio of the values of $F$ corresponding to two measured values of $\left(f / f_{0}\right)$ may be determined on the basis of field-intensity measurements with two loop antennas operating in the overlapping portion of their frequency ranges. Four such determinations were made using three loop antennas in each of two field-intensity measuring sets and are plotted in figure 2. Since, for each determination, loop antenna $A$ is operating near its lowest value of $\left(f / f_{0}\right)$ and loop antenna $B$ near its highest value, the ratio of the two field-intensity measurements should represent, to a first approximation, the value of $F_{B}$ corresponding to $\left(f / f_{0}\right)_{B}$. A closer value for $F_{B}$ may be obtained by taking a value for $F_{A}$ from any of the graphs of figure 2 corresponding to

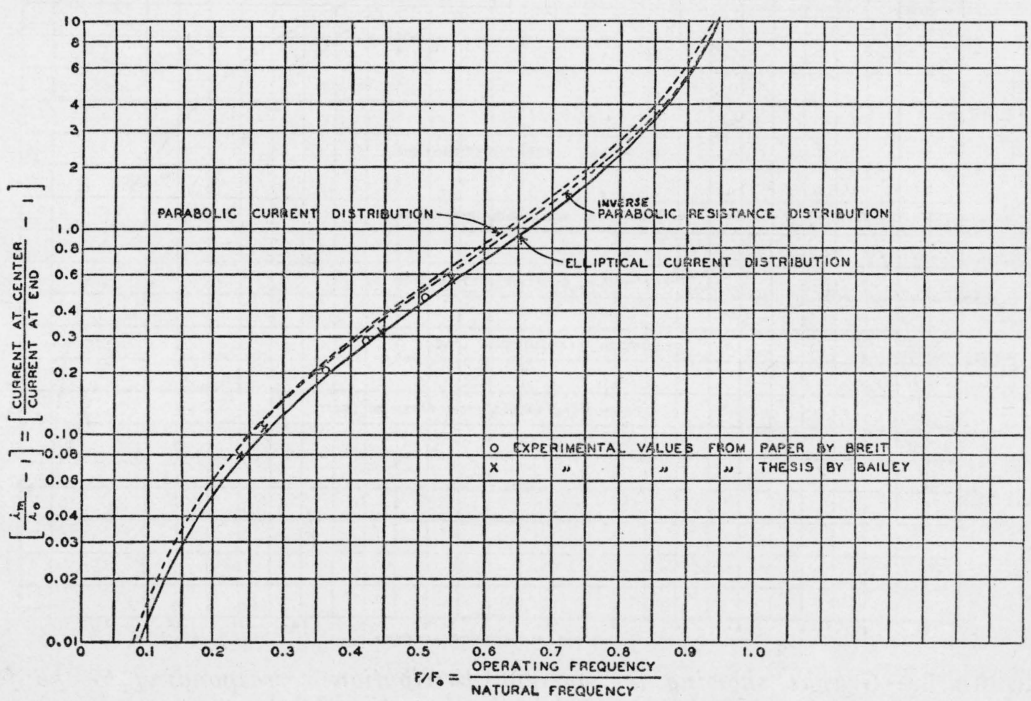

FIGURE 4.-Graphs showing the variation with frequency of the ratio of the current at the center of the loop antenna to the current at its end corresponding to three of the current distributions considered.

The open-circle points are from Breit's published values and the cross symbols denote values derived from Bailey's resistance measurements.

$\left(f / f_{0}\right)_{A}$ and increasing the ratio accordingly. In figure 2 the solid circles represent the results of the four determinations adjusted on this latter basis; the values of $F_{A}$ were in each case chosen from the graph corresponding to elliptical current distribution. The numerals in the illustration serve to identify the two points for each determination. In the first determination $(1-1)$, the ratio of measured field intensities was 1.068 and the values of $\left(f / f_{0}\right)$ for antennas $A$ and $B$ were 0.213 and 0.511 , respectively. From the graph, $F_{A}$ equals 1.013 . Hence the value for $F_{B}$ becomes $1.013 \times 1.068=1.082$. It will be noted that the experimental points agree very nicely with the theoretical graph. However, had the values of $F_{A}$ been chosen from one of the other theoretical graphs, the fit to that graph would not be nearly as good. 
The open circles in figure 2 represent the values of the correction factor $F$ at several measured values of $\left(f / f_{0}\right)$ for a commercial apparatus which employs a single loop antenna to cover the frequency range of from 550 to $1,550 \mathrm{kc} / \mathrm{s}$. (The correction factor was found to be very nearly the same for five sets of this manufacture.) The values were obtained from comparative field-intensity measurements with this apparatus and with a standard set corrected for the distributedcapacitance effect on the basis of overlapping frequency-range fieldintensity measurements. The experimental data are again seen to fit the theoretical graph for elliptical current distribution.

Added experimental evidence, to show that the graph corresponding to elliptical current distribution probably represents the true correction factor, will be presented in the following section.

\section{V. $\triangle C$ METHOD FOR ELIMINATING THE DISTRIBUTED- CAPACITANCE EFFECT}

It was first pointed out by Baker and Huxley ${ }^{4}$ that the true $Q$-factor of the antenna for a distributed induced voltage is obtained provided the measurement is carried out by the condenser-variation method. The reason for this is that the effective resistance measured in this method of determining the $Q$-factor is the average resistance throughout the loop antenna. In 1935 the Federal Communications Commission designed a field intensity measuring set for service as a secondary standard in its survey of clear-channel broadcast frequencies. For use in the conventional voltage-comparator method of measuring field intensity, the set embodied an oscillator, a number of interchangeable plug-in resistors and inductors, and thermoelements of different current ratings, for inserting known lumped voltages at the center of the loop antenna. The same means could be applied to calibrate the radio receiver as a sensitive electron-tube voltmeter. Hence, the $Q$-factor of each of the loop antennas, corresponding to lumped voltages at the center, could be measured. In addition, the apparatus included a small, calibrated, precision condenser, connected in parallel with the main tuning condenser; this $\Delta C$ condenser, in combination with the calibrated tube-voltmeter, permitted the measurement of the $Q$-factor by the condenser-variation method to a high degree of accuracy. Assuming that the latter determination yields the true value of $Q$-factor for a distributed field, the ratio of the values obtained by the two methods of measurement should equal the value of $F$ corresponding to the particular value of $\left(f / f_{0}\right)$ for which the measurements were made.

To test the efficacy of the $\Delta C$ method for eliminating the distributedcapacitance effect, comparative field-intensity measurements were first made using the three loop antennas of the set (in pairs) at overlapping frequencies. The results showed that the effect was practically eliminated. Next, a series of measurements was made of the $Q$-factors for distributed and lumped voltages using the two methods just described. The measurements were made for the three antennas of the set for different values of the argument $\left(f / f_{0}\right)$. The ratios of the $Q$ factors agreed quite closely with the values given by the graph corresponding to elliptical current distribution in figure 2 .

1 W. G. Baker and L. G. H. Huxley, Correction to Field Strength Measurements with Loop Antennae. Radio Research Board of Australia Bulletin 47, Report 1, Melbourne (1931). 
Next, a series of measurements was made of the $Q$-factors by the condenser-variation method using both lumped voltages and distributed fields. For a given antenna and a given value of $\left(f / f_{0}\right)$, the $Q$-factor was found to be the same whether a lumped voltage or a distributed field was employed.

Finally, a series of comparative field-intensity measurements was made using both the voltage comparator and the $\Delta C$ methods in the Federal Communication Commission's set, and also a standard set of the National Bureau of Standards; the latter also had incorporated in it means for measuring intensity by the voltage-comparator method. The results of the intercomparisons are shown in figure 5.

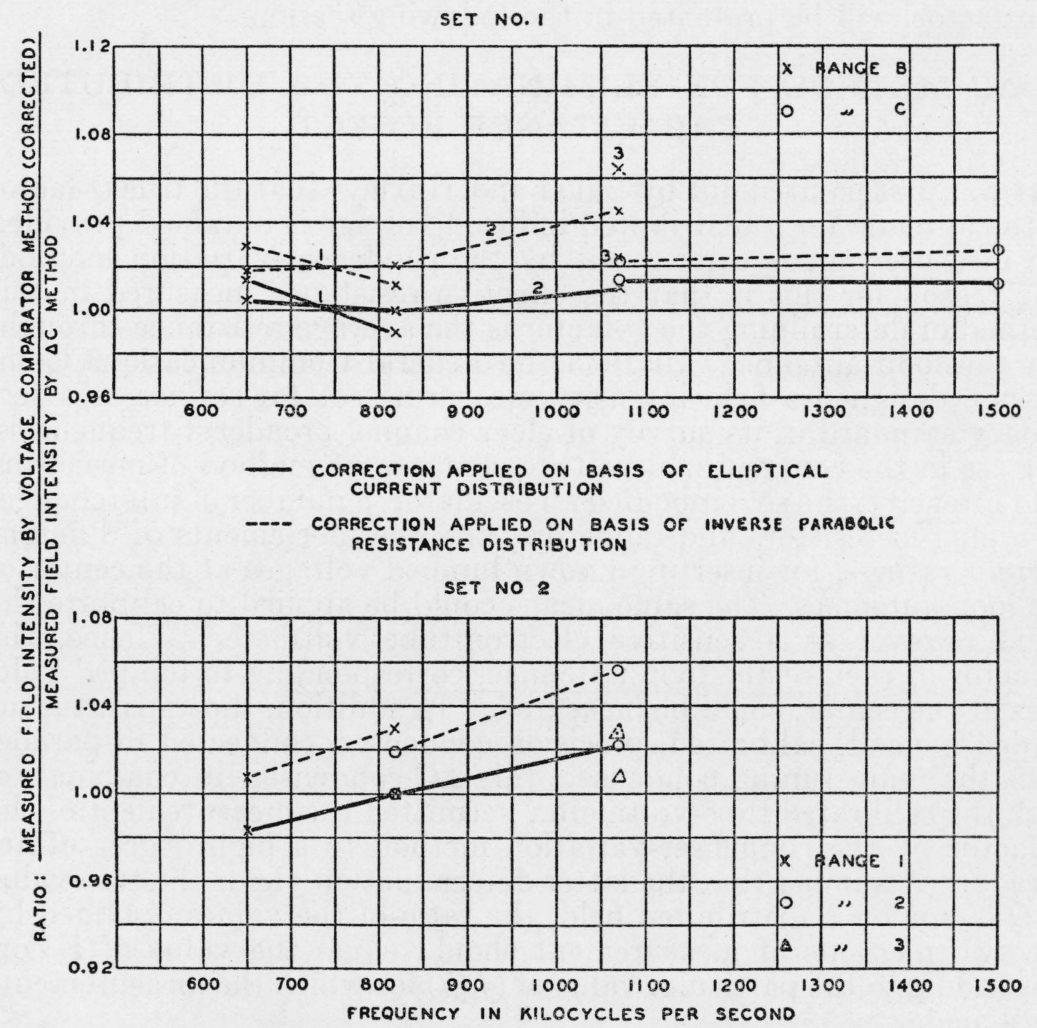

FIGURE 5.-Graphs showing the comparison of measured values of field intensity by the $\Delta C$ and voltage-comparator methods, the latter values being corrected for the distributed-capacitance effect by factors derived on the basis of elliptical current and inverse parabolic resistance distributions.

In the upper part of figure 5, the Bureau's standard set is compared against the $\Delta C$ measurements. The full lines plot the ratios of the field intensities measured with the Bureau's set, corrected in accordance with the factors derived for elliptical current distribution, to the field intensities measured by the $\Delta C$ method on the Commission's set. The cross symbols indicate that antenna $B$ was used on the Bureau's set and the open-circle symbols that antenna $C$ was used. The 
numbers near the lines indicate, respectively, that antenna 1 , 2 , or 3 was used on the Commission's set. It will be observed that the agreement was within 2 percent, the limit of accuracy of the measurements.

The dotted lines in figure 5 plot the corresponding ratios of the measured field intensities on the basis that the corrections to the measured values with the Bureau's set are made in accordance with the factors derived for inverse parabolic resistance distribution. (See fig. 2.) This distribution was chosen for a comparison since it represented the least departure from the theoretically correct elliptical current distribution. It will be seen that the agreement is not as good, one point being out by about 6 percent. If no corrections had been applied to the measurements with the Bureau's set, differences up to 6 percent (in the opposite direction) would have been obtained.

In the lower part of figure 5 the measurements with the voltagecomparator and $\Delta C$ methods in the Commission's set are compared. The full lines plot the ratios of the field intensities measured with the voltage-comparator method (and corrected in accordance with the factors derived for elliptical current distribution) to the field intensities measured with the $\Delta C$ method; the agreement is within 2 percent. Here, again, the dotted lines plot the corresponding ratios of the measured field intensities with the corrections to the voltage-comparator measurements made in accordance with the factors derived for inverse parabolic resistance distribution. The deviation is again up to 6 percent. Had no corrections been applied to the voltagecomparator measurements, differences up to 5 percent (in the opposite direction) would have been obtained.

An explanation of the small errors observed for the distributedcapacitance effect in this intercomparison may be of interest at this point. Both the Bureau's and the Commission's sets were designed to limit this error by employing, respectively, two and three loop antennas for covering the frequency range used. In this way, the upper value of $\left(f / f_{0}\right)$ employed was kept reasonably low, below 0.5 and 0.4 , respectively.

It will be seen that all of the experimental evidence points to the fact that the $\Delta C$ method is completely free from the distributedcapacitance error.

\section{UNTUNED LOOP ANTENNA}

It is of interest to note that the effect of distributed capacitance of the loop antenna on the accuracy of field-intensity measurements may be avoided by the expedient of using an untuned loop antenna, since uniform current distribution is then obtained in the loop antenna, so that the correction factor given by eq 9 is unity. There is, of course, the attendant disadvantage of required high receiver amplification, high set noise, etc. A set embodying this feature was calibrated at the Bureau. The ratio of the measured values of field intensities with this set to the measured values with a standard set (after correction of the latter for distributed-capacitance effect) was found to equal $1.05 \pm 0.03$, in the frequency range of from 550 to $1,600 \mathrm{kc} / \mathrm{s}$. There was no evidence of a definite trend of variation of this ratio with frequency. Hence, assuming that the 5-percent average difference was caused by some other factor, not determined in the intercompari- 
son, the distributed-capacitance effect appears to be avoided with an untuned loop antenna.

\section{SHIELDED LOOP ANTENNA}

Another expedient for minimizing the distributed-capacitance effect is to employ a shielded loop antenna. One terminal of the loop
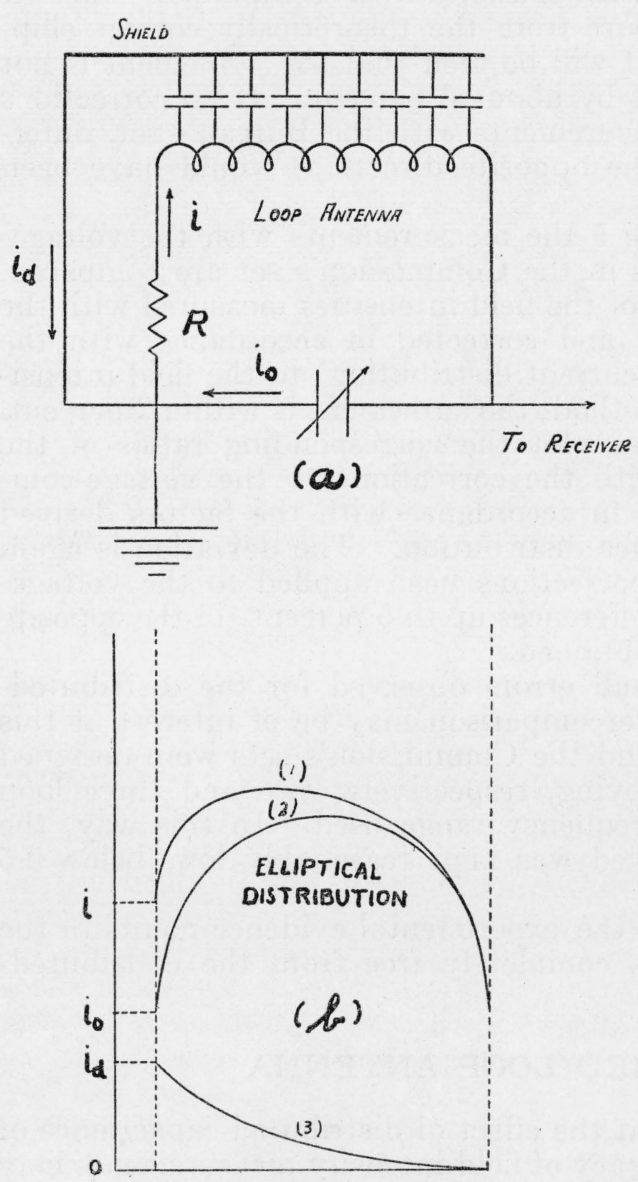

TURNS ALONG LOOP ANTENNA

FIGURE 6.-Sketch and graphs showing the current distribution in an unbalanced shielded loop antenna having the calibrating voltage inserted at its grounded end.

independent of the distribution of voltage, the current distribution of graph 1 will apply for both the distributed field voltage and the lumped calibrating voltage. An analysis of why this type of distribution tends to minimize the effect of the distributed capacitance of the loop antenna follows: ground through the resistor across which the calibrating voltage is applied. The shield and one side of the tuning condenser are grounded at the same point. The other terminal of the loop antenna and the other side of the tuning condenser are connected to the receiver input. (See fig. 6(a).) The calibrating voltage is thus applied in series with the loop antenna at its grounded end. The current charging the distributed capacitance between the loop antenna and the shield is seen to pass through the calibrating resistor.

E. The current distribution along the turns of the loop antenna now becomes approximately as shown by graph 1 of figure 6 (b). The current in any turn may be considered as being made up in part by the current which would exist if the loop antenna were unshielded and balanced with respect to ground (graph 2) and in part by the charging current for the effective distributed capacitance to the shield of the portion of the loop antenna beyond the turn considered (graph $3)$. Since the current distribution at resonance is

antenna is connected to 
Proceeding as in the derivation of eq 9 , the current $i_{0}$ through the tuning condenser, which is produced by the voltage $e$ across the calibrating resistor, is

$$
i_{0}=\frac{e}{\sqrt{R_{0} R}}
$$

where $R_{0}$ and $R$ are, respectively, the equivalent resistances of the loop antenna at the ungrounded and grounded ends. Hence, when $e$ is adjusted to give the same value of $i_{0}$ as produced by the field voltage (see eq 4), it has the magnitude

$$
e=\int e_{x} \sqrt{\frac{R_{0}}{R_{x}}} \cdot d x
$$

Hence, when the assumption is made that $e=\int e_{x} \cdot d_{x}$, the evaluated field intensity is too high and must be corrected by a factor which comes out to be equal to

$$
F=\frac{i}{i_{a v e}} .
$$

Here $i$ is the current through the calibrating resistor and is greater than the current through the condenser. (See Fig. 6.) Since the current $i$ may be equal to the average current along the loop antenna (if the equivalent distributed capacitance between the loop antenna and the shield is of the correct magnitude) the correction factor, as given by eq 16, may be equal to unity. Furthermore, since the current distribution is a function of the relative magnitudes of two distributed capacitances, the condition of unity correction factor may prove to be independent of the operating frequency.

It is of interest to note that the tendency to minimize the distributed-capacitance effect is produced solely by the unbalanced connection of the loop antenna with respect to ground and not by the shield. The latter controls the value of the current $i_{d}$ and its distribution and hence determines how closely the condition of unity correction factor is approached. The shield, however, serves the important function of eliminating the vertical or antenna effect. For the case where the loop antenna is balanced and the calibrating voltage is inserted at one end, the currents at the two ends of the loop antenna are equal $\left(i=i_{0}\right)$. The current distribution along the loop antenna for this balanced condition is given by eq 12 . Substituting these two relationships in eq 16 , we have

$$
F=1-\left(\frac{f}{f_{0}}\right)^{2},
$$

which is the correction factor for the calibrating voltage inserted at one end of a balanced loop antenna. Here the factor is seen to vary with frequency. See, for comparison, the expressions for the correction factor given in table 1 for the case where the calibrating voltage is inserted at the center of the loop antenna.

The action of the antenna unbalance in minimizing the distributedcapacitance effect was determined in the calibration of a field-intensity measuring set having a shielded loop antenna with the calibrating 
voltage applied as indicated in figure 6(a). A switch in the loop antenna permitted using either a 16-turn or a 12-turn connection, as desired. The object of the calibration was to determine the loop-antenna coefficients for the two connections. The results of the calibration are given in table 2. The first column gives the operating frequency, the second column the ratio of the operating frequency to the natural frequency, and the third column the corresponding measured values of the antenna coefficient. The latter is defined in terms of the field intensity, the operating frequency and readings of the output meter and voltage attenuator of the set; it constitutes a lumped factor including the value of the calibrating voltage and the turns area of the antenna. The fourth column of table 2 gives the value this factor would have for a single-turn loop antenna of the same dimensions. The fifth column gives the percentage deviation of the individual determinations from the average measured value. The trend of variation of the antenna coefficient with frequency is seen to be quite small, whereas the operating frequencies employed would appear to require a material correction factor (see eq 17) were it not for the unbalanced current distribution. However, such trend as is noted points to the importance of checking individual designs for possible variation of the antenna coefficient with frequency.

TABLE 2.-Data showing negligible distributed-capacitance effect for shielded loop antenna

\begin{tabular}{|c|c|c|c|c|}
\hline $\begin{array}{l}\text { Frequency } \\
(f)\end{array}$ & $\begin{array}{l}\text { Ratio of } \\
\text { operating } \\
\text { frequency } \\
\text { to natural } \\
\text { frequency } \\
\left(\frac{f}{f_{0}}\right)\end{array}$ & $\begin{array}{c}\text { Loop- } \\
\text { antenna } \\
\text { coefficient } \\
\left(K=\frac{F f}{M A}\right)\end{array}$ & $\begin{array}{l}\text { Equivalent } \\
\text { loop-an- } \\
\text { tenna coef- } \\
\text { ficient for } \\
\text { one turn } \\
(K N)\end{array}$ & $\begin{array}{l}\text { Percentage } \\
\text { deviation } \\
\text { from aver- } \\
\text { age value } \\
\text { of } K N\end{array}$ \\
\hline \multicolumn{5}{|c|}{ 16-TURN CONNECTION OF LOOP ANTENNA } \\
\hline $\begin{array}{r}k c / s \\
650 \\
650 \\
800 \\
850 \\
1,050 \\
1,050 \\
1,100\end{array}$ & $\begin{array}{l}0.29 \\
.34 \\
.42 \\
.45 \\
.55 \\
.55 \\
.58\end{array}$ & $\begin{array}{l}424 \\
425 \\
415 \\
414 \\
428 \\
436 \\
428\end{array}$ & $\begin{array}{l}6,780 \\
6,800 \\
6,640 \\
6,625 \\
6,845 \\
6,975 \\
8,845\end{array}$ & $\begin{array}{r}-0.2 \\
+.1 \\
-2.2 \\
-2.5 \\
+0.8 \\
+2.7 \\
+0.8\end{array}$ \\
\hline \multicolumn{5}{|c|}{ 12.TURN CONNECTION OF LOOP ANTENNA } \\
\hline $\begin{array}{l}1,050 \\
1,100 \\
1,300 \\
1,400 \\
1,500\end{array}$ & $\begin{array}{l}0.42 \\
.45 \\
.52 \\
.56 \\
.61\end{array}$ & $\begin{array}{l}568 \\
552 \\
564 \\
575 \\
575\end{array}$ & $\begin{array}{l}6,820 \\
6,630 \\
6,750 \\
6,900 \\
6,900\end{array}$ & $\begin{array}{l}+0.4 \\
-2.4 \\
-0.6 \\
+1.7 \\
+1.7\end{array}$ \\
\hline
\end{tabular}

\section{PRECAUTIONS FOR ATTAINING MAXIMUM ACCURACY}

In section II an estimate was given of the probable absolute accuracy of commercial field-intensity measuring sets at frequencies below about $1,600 \mathrm{kc} / \mathrm{s}$. It was shown that by applying corrections for the several errors likely to occur in the different methods, the absolute 
accuracy may be stepped up from not better than 15 or 20 percent to a possible 5 percent. Except for errors of types (g) and (h), the errors encountered are all amenable to correction on the basis of laboratory measurements. As was shown, errors of type (h) may be taken into accurate account by measuring the natural frequency of the loop antenna and applying a theoretical correction factor as a function of the argument $\left(f / f_{0}\right)$. Hence, where care is taken to minimize possible distortion of the field which it is desired to measure, measurements may, in general, be carried out to the 5-percent accuracy

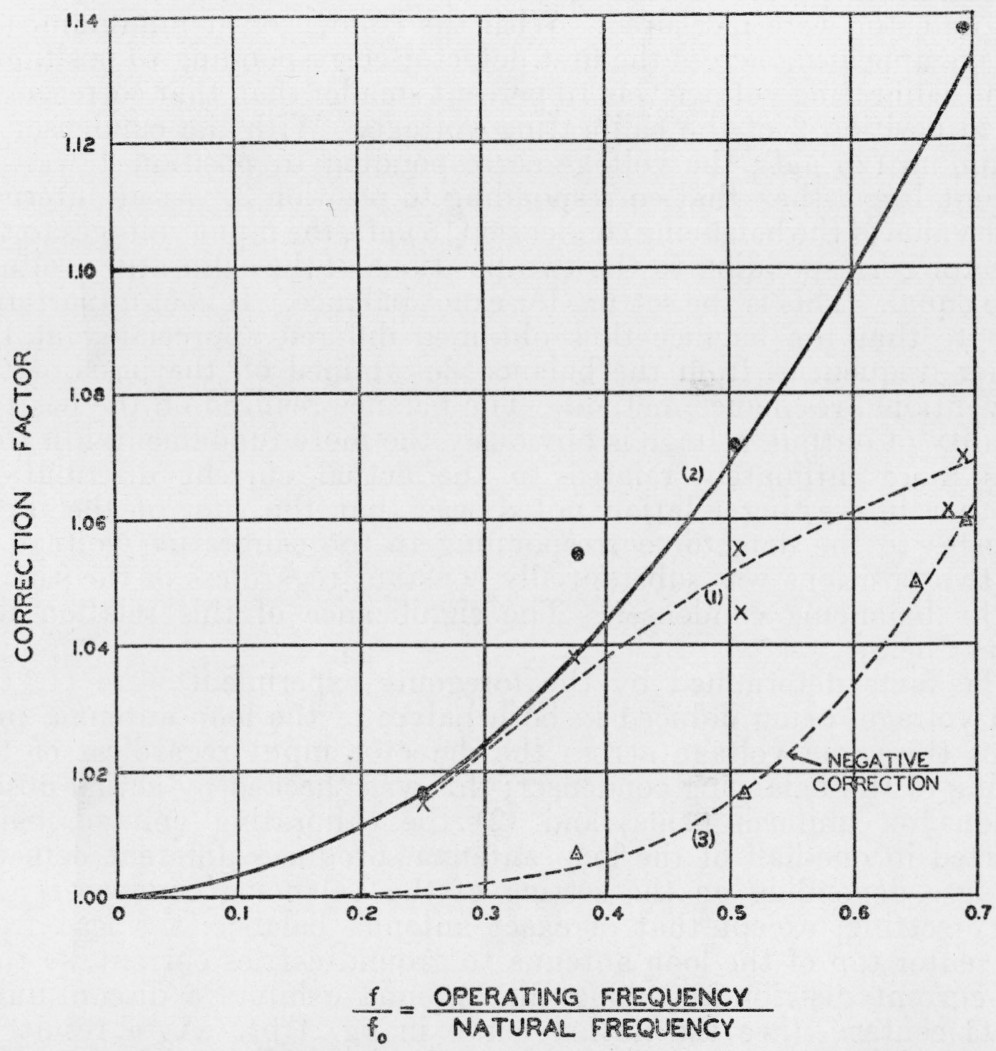

FIGURE 7.-Graphs showing partial neutralization of the distributed-capacitance effect by an error introduced due to antenna unbalance.

In the derivation of the correction factor to be applied for the distributed-capacitance effect when the lumped calibrating voltage is inserted at the center of the loop antenna, it was assumed that the loop antenna was in exact balance. The effect of incorrect adjustment of the balancing condenser (see fig. 1) will now be considered.

In one field-intensity measuring set, the variation of the measured values of the correction factor as a function of $\left(f / f_{0}\right)$ was found to be as shown by graph 1 of figure 7. The cross symbols represent the actual observations. The cause for the large departure of the measured values of the correction factor from the values derived theoretically (reproduced as graph 2) was investigated and was found to be 
unbalance of the loop antenna. The balancing condenser was found to be set at its minimum value. Upon adjusting it for exact balance (at $1,500 \mathrm{kc} / \mathrm{s}$ ) the correction factors for several values of $\left(f / f_{0}\right)$ were again determined and are given by the solid-circle symbols in figure 7 . Note the excellent agreement with the theoretically derived factors.

The method employed in adjusting the balancing condenser for exact antenna balance seemed to indicate the reason for the difference between graphs 1 and 2. Referring to figure 1, the calibrating voltage was inserted alternately at points 1 and 2 for different settings of the balancing condenser and the corresponding voltages applied to the first detector were measured. With the condenser at minimum, the voltage appearing across the first detector corresponding to position 1 of the calibrating voltage was 10 percent smaller than that corresponding to position 2 of the calibrating voltage. With the condenser at maximum $(25 \mu \mu \mathrm{f})$, the voltage corresponding to position 1 was 15 percent larger than that corresponding to position 2. At an intermediate value of the balancing condenser ( $15 \mu \mu \mathrm{f})$, the input voltages to the detector corresponding to the two positions of the calibrating voltage were equal. This is the setting for exact balance. It is of importance to note that the balance thus obtained differed appreciably at the higher frequencies from the balance determined on the basis of the conventional resonance method. The balance realized on the basis of equality of output voltage is obviously the more fundamental in that it is more intimately related to the actual current distribution. Another interesting relation noted was that the sum of the input voltages to the detector corresponding to the calibrating voltage at the two positions was substantially constant regardless of the setting of the balancing condenser. The significance of this relation will appear below.

The facts determined by the foregoing experiment were (1) the field voltage, being induced in both halves of the loop antenna, produces the same voltage across the detector input regardless of the setting of the balancing condenser; this was checked by actual observations on uniform fields; and (2) the calibrating voltage, being inserted in one-half of the loop antenna, produces different detector voltages depending on the setting of the balancing condenser. At every setting, except that of exact antenna balance, the lead from the center tap of the loop antenna to ground carries current, so that the current distribution along the antenna exhibits a discontinuity at its center. (See the dotted curve in fig. 1(b). As a result an error is introduced by antenna unbalance (additional to the distributed capacitance error) in equating the calibrating voltage to the induced field voltage. This error may be either positive or negative depending on the setting of the balancing condenser and upon the point of insertion of the calibrating voltage.

In the case considered in graph 1 of figure 7, the balancing condenser being set at minimum and the calibrating voltage inserted at position 1 , the voltage produced across the detector was less than would have been produced were the condenser at its proper setting. Hence, a negative error was introduced which served to offset, in part, the distributed-capacitance effect. It is obvious that the magnitude of this error varied not only with the degree of antenna unbalance but also with the magnitude of the balancing and tube capacitances in 
relation to the tuning condenser and, hence, with $\left(f / f_{0}\right)$. In figure 7, graph 3 was obtained by dividing the points on graph 2 by the corresponding points on graph 1 and should, therefore, show the variation of the error caused by unbalance as a function of $\left(f / f_{0}\right)$. The triangle symbols correspond to direct measurements in this error for several values of $\left(f / f_{0}\right)$. Each measurement consisted in first ascertaining the balance condition in the manner described and then, with the calibrating voltage at position 1 , determining the ratio of the voltages produced across the first detector corresponding to balance and minimum setting of the balancing condenser. The agreement with graph 3 is quite good.

Two important conclusions may be drawn from the experiments just outlined. First, it is important to obtain exact antenna balance if the derived correction factors for the distributed-capacitance effect are to be applied. Secondly, a deliberate unbalance of the loop antenna may be introduced to offset automatically a major part of the distributed-capacitance effect. When combined with the use of antennas operating at values of $\left(f / f_{0}\right)$ less than about 0.5 , the resultant error may be reduced to the order of a few percent. The agreement of graph 3, which was derived indirectly from two sets of field-intensity measurements, with the laboratory determinations of the error due to antenna unbalance (indicated by the triangle symbols) points to the fact that any vertical or antenna effect introduced by the unbalance had little influence upon the measured field intensities.

To attain maximum accuracy, careful consideration must also be given to possible field distortion (i. e., error (g)). As already noted in section II, errors up to 10 percent were detected because of the distortion of the field by the set container, while errors up to 20 percent were observed by distortion of the field by the car in which the set was installed. Since the distortion is produced by secondary fields radiated from objects placed in the primary field, it is important to keep such objects as small as possible. There is, therefore, a theoretical argument for keeping the set container small, in addition to the practical one of portability. Where it is essential to have a large container or to install the set on an automobile, the loop antenna should be mounted as far as possible above the disturbing metal.

Figure 8 shows the effect of a large set container and the possibility of securing true measurements by placing the antenna outside of the portion of the field which is distorted. At the top of figure 8 is shown the variation in the measured field as a function of the orientation of the loop antenna with respect to the minor axis of the set container. The true value of the field is measured when the plane of the loop antenna coincides with the minor axis ( 0 to 180 degrees); the measured value is some 10 percent too high when the plane of the loop antenna is along the major axis (90 and 270 degrees). In the middle of figure 8 is shown the variation in the measured field when a small coil antenna is used in place of the loop antenna. The coil, being much nearer to the set container, is within the distorted field caused by the container; hence the error corresponding to the 90 - and 270-degree positions is some 25 percent. There is seen to be practically no frequency effect in the amount of field distortion. When an extension fitting, approximately 18 inches long, was used to raise the coil antenna above the set, the true measured value was obtained for all orientations of the 
coil with respect to the set. See the graphs at the bottom of figure 8 . Obviously, the same treatment may be applied to the mounting of a loop antenna on an automobile, although the height required above the car top may introduce practical difficulties.

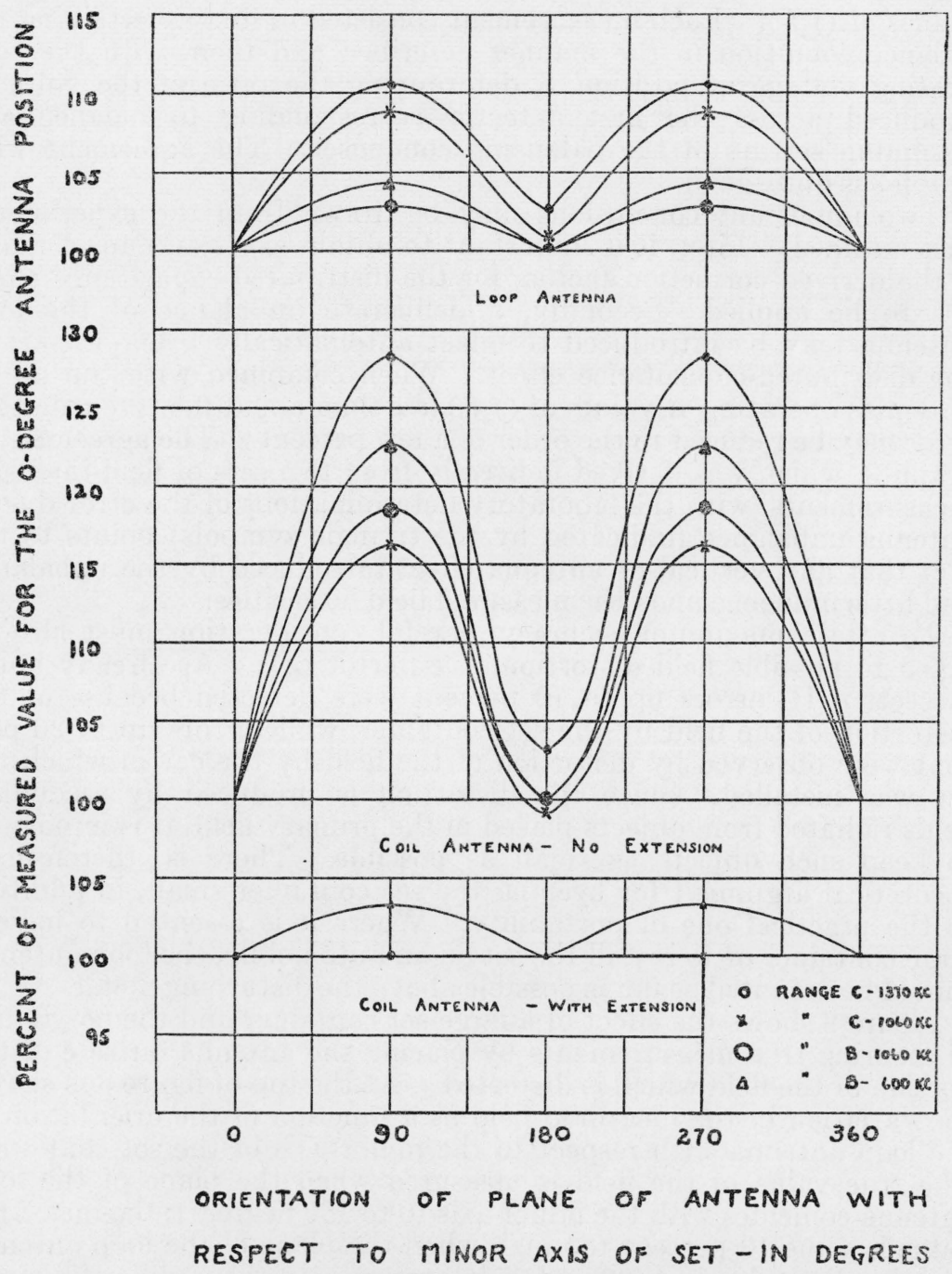

FIGURE 8.-Graphs showing the effect of distortion of the field by the set container upon the accuracy of field-intensity measurements.

Removal of the measuring antenna from the distorted field results in true field measurements.

As a final point of interest, figure 9 shows the results of calibration of a set which exhibited errors having a marked variation with frequency. The set was mounted in a car, but since no measurements were made with it outside of the car, there was no evidence to indicate whether or not the errors were due to the car installation. However, 
other causes for the variation of the error with frequency may include set regeneration, resonant circuits in the connecting leads from the loop antenna to the set, and induction in the loop antenna from the calibrating oscillator. The observations on this set indicate the importance of preliminary analysis for possible errors before embarking upon actual field-intensity measurements.

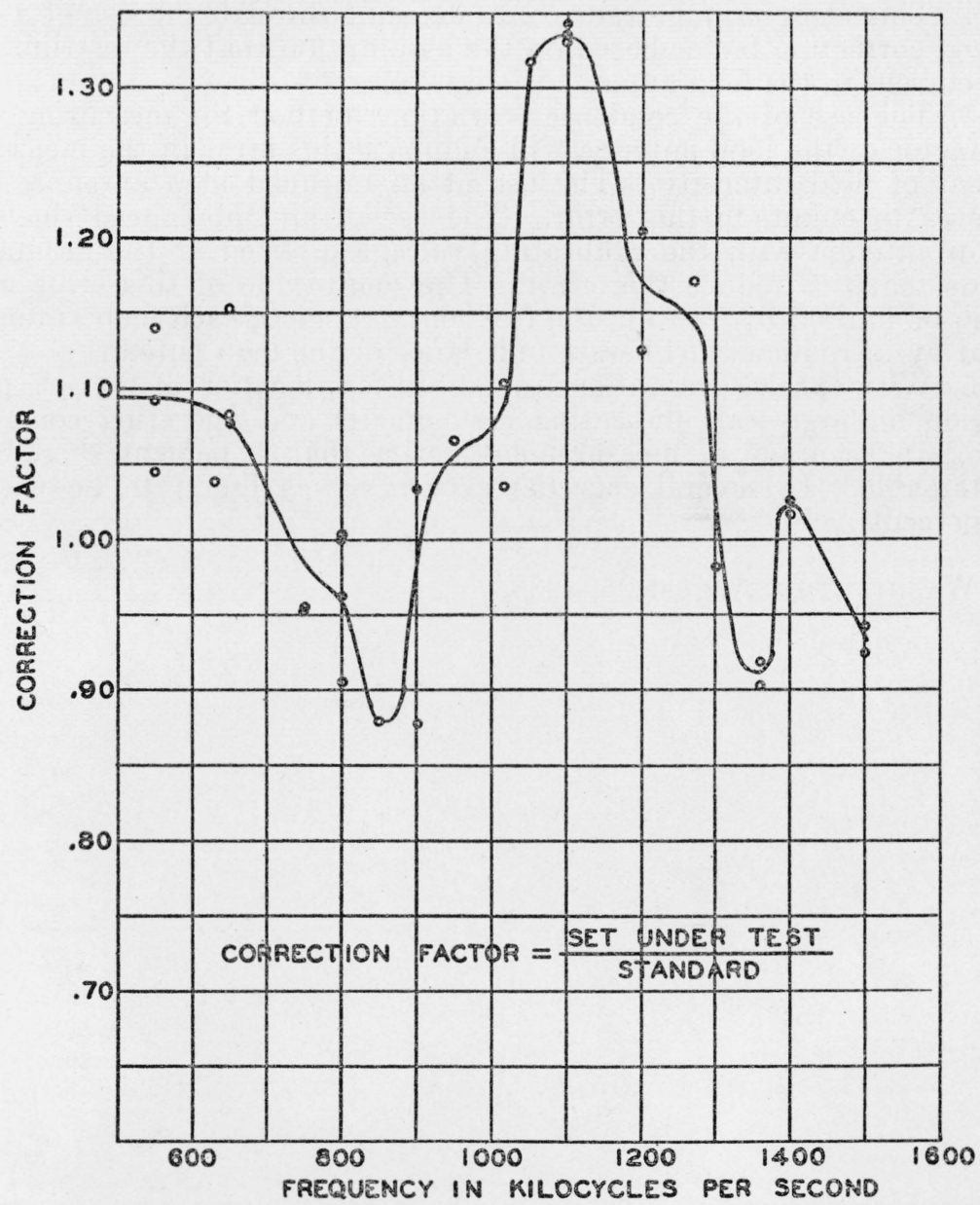

FIGURE 9.-Calibration of a set showing marked variation of the errors as a function of the operating frequency.

\section{CONCLUSIONS}

Summarizing the data presented in this paper, the following conclusions may be drawn.

1. Commercial equipments designed for field-intensity measurements at frequencies below $1,600 \mathrm{kc} / \mathrm{s}$ provide absolute accuracies not greater than 20 percent unless special precautions are taken to correct for the errors enumerated in section $I$. 
2. Of the errors listed, some are common to all the methods of measurement, while others pertain to specific methods. Of the methods discussed, the voltage-comparator arrangement is subject to the least number of errors in the frequency range considered.

3 . The error introduced by virtue of the distributed capacitance of the loop antenna is often the most serious of the several errors. Its magnitude may be up to 15 percent in certain sets.

4. This error may be taken into account through the use of a derived correction factor based on the assumption that the distribution of current in the loop antenna is elliptical.

5. The use of the condenser-variation method for measuring the $Q$-factor of the loop antenna will eliminate this error in the measurement of field intensity. The use of an untuned loop antenna also serves to eliminate this error. The use of an unbalanced shielded loop antenna with the calibrating voltage inserted at the grounded ends tends to reduce this error. The magnitude of this error may also be limited by limiting the frequency range of each loop antenna, and by introducing deliberate unbalance of the loop antenna.

6. With special precautions in calibration, location of the set, provision of large-scale indicating instruments and operating controls, etc., an accuracy of measurement better than 5 percent should be attainable. In several sets the accuracy was found to be within 2 percent.

Washington, August 25, 1938. 